\title{
Formation au prototypage de systèmes électroniques 2D et 3D Initiation à la Plastronique 3D et aux dispositifs MID
}

\author{
Ph. Lombard ${ }^{1}$, V. Semet ${ }^{1}$, M. Cabrera ${ }^{2}$ \\ (Philippe.Lombard@univ-lyon1.fr) \\ ${ }^{1}$ Maitre de conférences, ${ }^{2}$ Chargé de recherche \\ Université Claude Bernard Lyon1 (UCBL), Institut des Nanotechnologies de Lyon (INL), \\ UMR 5270, Université Lyon 1, \\ Bât. L. Brillouin, 43 Bd du 11 Nov. 1918, F69622 Villeurbanne, France.
}

\begin{abstract}
Résumé
Nous présentons dans ce papier un projet de formation innovant relatif au prototypage de circuits et systèmes électronique 2D et 3D. Les apprenants seront initiés aux technologies de ruptures que sont la plastronique et les circuits imprimés 3D sur support rigide (Molded Interconnect Device ou Dispositifs Interconnectés Moulés). Ces dernières nécessitent d'acquérir des compétences aussi bien dans le domaine de la plasturgie que dans celui de l'électronique. Alors que la technologie et l'innovation se situent aux interfaces de ces domaines, il est aujourd'hui impératif de casser les modes de conceptions (usuellement en silo) et de repenser la fiche de route (roadmap). Seule une avancée conjointe permettra d'apporter et d'engranger un savoir-faire innovant pour converger vers des solutions pertinentes et une implémentation optimale. Nous souhaitons aller vers la création d'une plateforme dédiée. Cette dernière mutualisera des moyens techniques et humains et affichera une bonne visibilité. Le tissu industriel de la région Rhône-Alpes issue d'une longue histoire est à ce titre, particulièrement propice à l'émergence de cette action. Aujourd'hui, un groupe de travail associe des acteurs industriels et académiques avec pour objectif de mettre en place cette formation multi-physiques et multi-domaines alliant électronique, plasturgie, mécatronique...
\end{abstract}

Mots-clés : Prototypage 2D et 3D, plastronique, plasturgie, MID, DIM, carte électronique, circuit imprimé, $P C B$, formation, plateforme technologique.

\section{INTRODUCTION}

Dans le contexte de mondialisation actuel, l'industrie des nouvelles technologies doit sans cesse innover, s'adapter et faire face à une concurrence de plus en plus féroce. Seuls les produits innovants à forte valeur ajoutée permettront aux entreprises et industries françaises de perdurer et de se développer sereinement.

Aussi, un effort important doit être consacré à la R\&D. Par ailleurs, une meilleure formation des employés (cadres, techniciens) et collaborateurs est indispensable.

Ce projet porte sur la création d'une formation au prototypage de systèmes électroniques $2 \mathrm{D}$ avec une initiation et une sensibilisation à la Plastronique 2D et 3D. L'enseignement reposera sur une base scientifique théorique solide, couplée à une forte expertise pratique (en réponse aux recommandations et enjeux préconisés dans [1-3]). La création d'une plateforme dédiée et commune garantira le succès de celle-ci.

\section{ENVIRONNEMENT DE L'ACTION}


Les technologies de conception et de câblage des circuits électroniques ont subi de profondes transformations ces dernières années. La miniaturisation, l'augmentation de la densité d'intégration et la baisse des coûts de production ont entraîné le remplacement progressif des technologies traditionnelles «traversantes » THT (Through Hole Technology) par des technologies de surfaces CMS (Composants Montés en Surface) qui sont aujourd'hui incontournables.

Nous illustrons, sur la figure 1, l'évolution des technologies dans la fabrication de circuits électroniques.

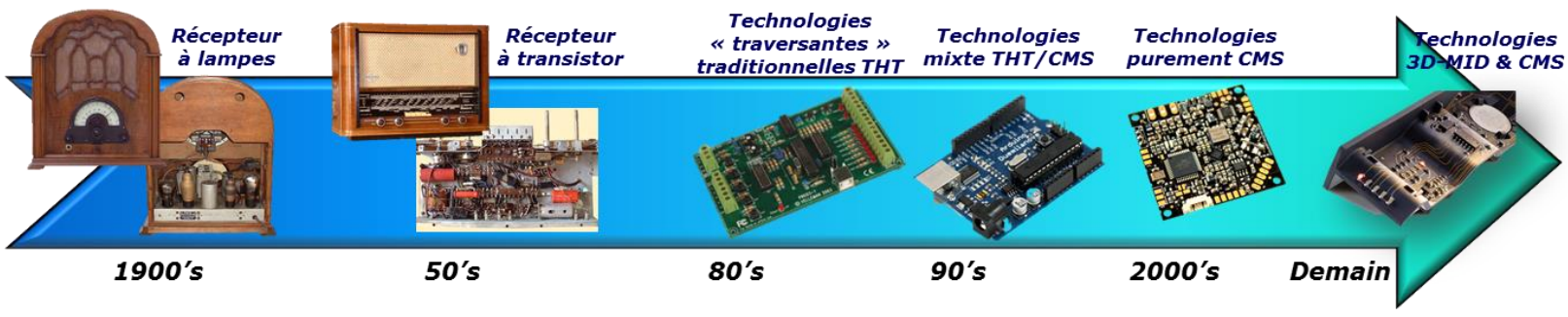

Fig. 1 : Evolution des technologies de câblage des cartes électroniques (banque d'images web).

Par ailleurs, le développement de nouvelles technologies permet l'émergence de systèmes plastiques intelligents. Ces systèmes associent des composants électroniques (apport de l'intelligence) sur des pièces plastiques (apport des fonctions mécaniques et du packaging), qui sont souvent des films en polymère. Une nouvelle industrie est en émergence : la Plastronique. Outre toutes les applications liées aux cartes à puce, citons par exemple : l'électronique organique, souple, étirable $^{1}$ (Fig. 2). Nous constatons aussi un renouveau dans la recherche sur les procédés avec des méthodes de fabrication non conventionnelles en électronique comme l'impression par jet d'encre, la sérigraphie, l'imprimerie (avec ses nombreuses variantes : flexographie, lithographie, offset...), etc.

(a)

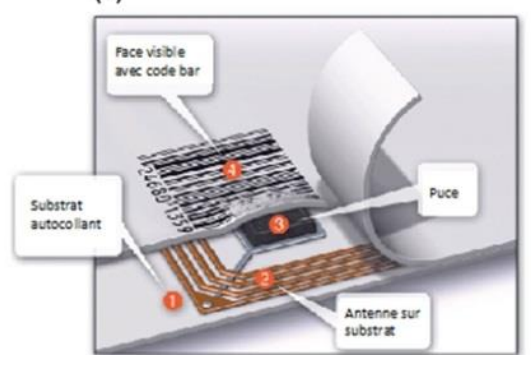

(b)

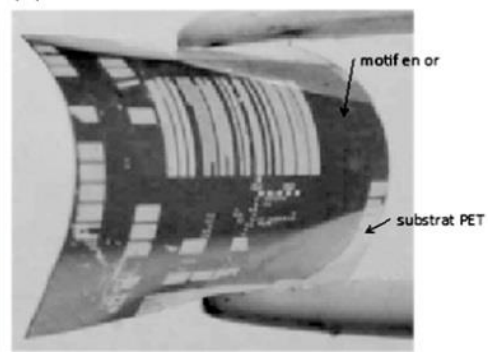

(c)

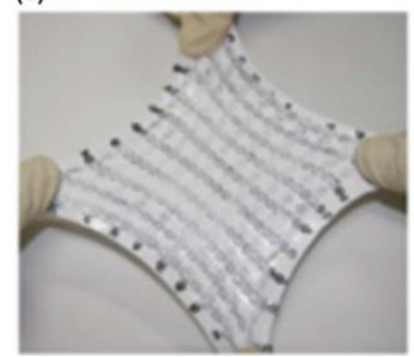

Fig 2 : (a) Tag RFID composé d'une puce silicium et d'une antenne (http://skyrfid.com/RFID_Item_Tag.php).

(b) Motifs en or sur un substrat en polymère polytéréphtalate d'éthylène (PET) pour l'électronique organique

(A. Benor et al. Org. Electron. 2010). (c) Transistors organiques déposés sur un substrat étirable en polydimethylsiloxane (T. Sekitani et al. Adv. Mater. 2010).

Une classe nouvelle d'objets plastroniques est constituée par les MIDs, acronyme anglais pour Molded Interconnect Device, que nous désignerons ci-dessous par le terme français DIMs pour Dispositifs Interconnectés Moulés.

Les DIMs sont des circuits imprimés 3D sur polymère rigide. Un DIM est un système mécatronique constitué d'un support $3 \mathrm{D}$ en polymère sur lequel le dispositif électronique est directement disposé ; les pistes métalliques épousant la forme du substrat sur lesquelles seront ensuite reporter les composants. Il est possible ainsi d'imbriquer étroitement des parties électroniques, mécaniques et optiques..., ce qui permet d'intégrer de nouvelles fonctions ou de mieux intégrer des fonctions existantes, de réduire le nombre d'étapes d'assemblage, de simplifier le dispositif, d'améliorer la fiabilité, etc.

Comme montré à la figure 3 , les DIMs sont déjà produits à grande échelle, notamment pour réaliser des antennes de smartphones ainsi que pour d'autres applications industrielles, comme dans

\footnotetext{
${ }^{1}$ Il existe aussi une électronique sur papier, sur textile, etc.
} 
l'automobile. D'innombrables nouveaux usages peuvent être envisagés dans des domaines tels que les procédés industriels, l'instrumentation, l'aérospatial, l'énergie, la domotique, les objets connectés...).

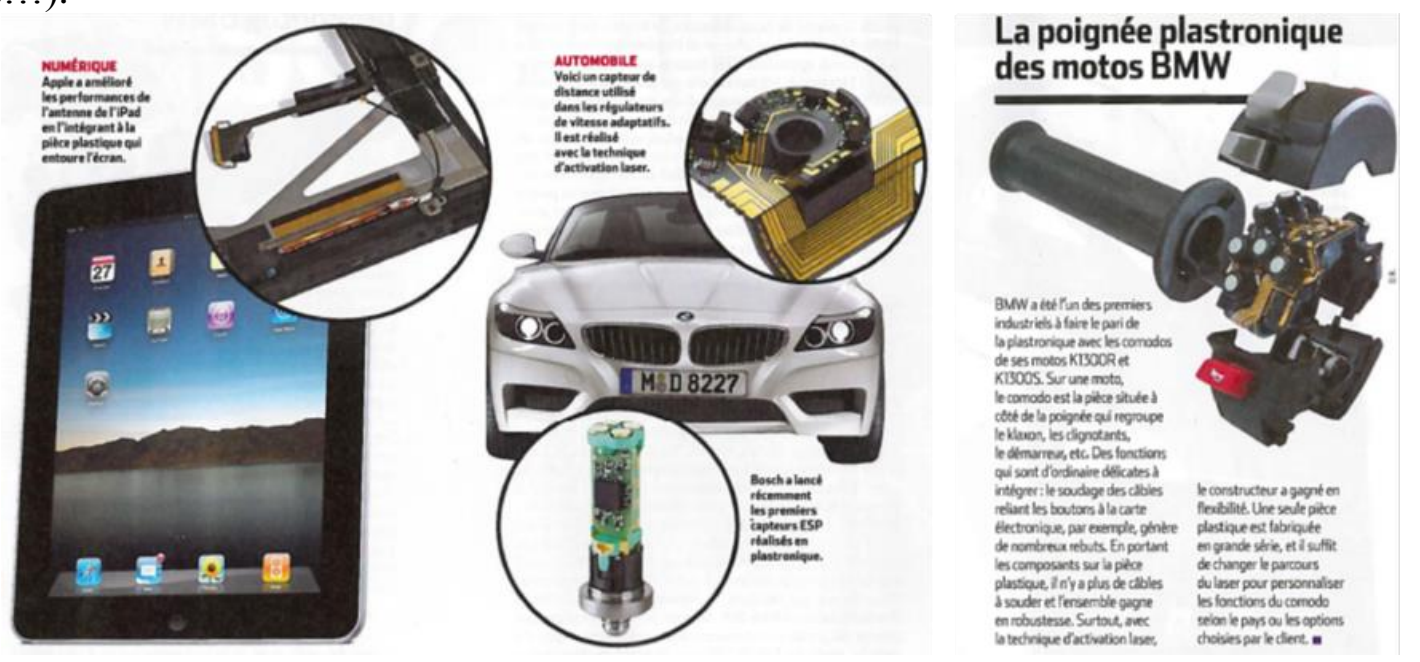

Fig. 3 : Exemples de DIMs produits en masse

(article de l'Usine Nouvelle de mai 2012)

Plusieurs technologies sont aujourd'hui disponibles pour réaliser ces objets : bi-injection, ablation laser et surtout un procédé très flexible qui a démocratisé la technologie : la structuration laser. De son côté, l'INL développe sa propre technologie d'élaboration de DIMs par micro-tamponnage.

Par ailleurs, indépendamment des méthodes de fabrication, les activités de recherche portent sur des problématiques plus globales liées à l'implémentation de fonctions électriques, de communication, mécaniques, fluidiques, optiques, thermiques etc. Un effort considérable est fait afin de repenser l'ensemble des méthodes de conception et d'implémentation (roadmap, CAO 3D électronique/mécanique...) ainsi que pour développer les outils de production (dépose des composants, brasage...) et de tests nécessaires au développement de ces technologies nonconventionnelles.

Les DIMs permettent de laisser libre cours à notre créativité dans la conception de systèmes et notamment de tirer profit de la géométrie 3D du support. En plus d'un éventuel empilement successif de couches et fonctions électroniques, le positionnement des fonctions et composants électroniques peut être fait dans l'espace en alliant les éventuelles propriétés d'élasticité et de flexibilité des supports (Fig. 3). Il est possible par exemple de réaliser des antennes électromagnétiques par technologie DIM avec des orientations différentes dans l'espace.
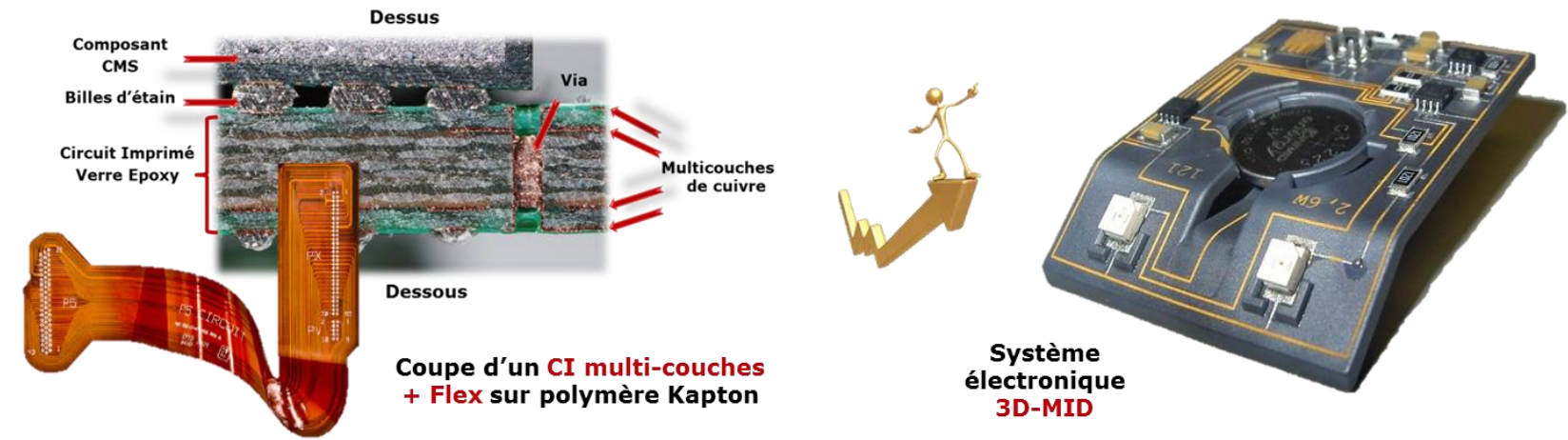

Fig. 4 : Coupe d'un Circuit Imprimé - CI multicouches + circuits empilés (BGA, flip-chip...), circuit souple (Flex) et circuit 3D-MID (banque d'images web). 
Dans tous les cas, quel que soit le mode de conception, la réalisation de circuits électroniques (tout comme les substrats polymères) exige de respecter de nombreuses règles et protocoles. Le coût des équipements et moyens techniques à mettre en place, mais plus encore, le manque criant et récurrent d'information et surtout de formation nécessitent de proposer un enseignement adapté. Ainsi, seul un travail conjoint et transversal permettra à terme d'anticiper et d'optimiser au mieux les produits de demain :

«Les objets de demain doivent, dès le début, être pensés comme étant un «tout ». L'électronique doit épouser formes et contours pour s'intégrer le plus justement possible. Designer, électronicien et intégrateur doivent dès lors travailler de pair. "

Dans la formation que nous comptons mettre en place, nous proposons de présenter les bases théoriques et pratiques des technologies d'assemblage et de conception de cartes et systèmes plastroniques. Le but est d'intégrer les technologies d'aujourd'hui afin de comprendre les celle de demain (plastronique et DIM). Cette formation comprendra un fort investissement pratique. L'idée est de répondre concrètement aux problématiques des acteurs du secteur via des activités de prototypage alliées à une formation théorique.

\section{DESCRIPTION DE L'ACTION}

Dans ce contexte, lors de l'élaboration d'un produit, les électroniciens, les plasturgistes et les mécaniciens ne peuvent plus travailler séparément et développer leurs applications sans se soucier des contraintes des autres acteurs du système.

Alors que la technologie et l'innovation se situent aux interfaces, il est aujourd'hui impératif de casser la conception en silo et le cloisonnement de l'organisation des formations.

Nous pouvons citer quelques défis et enjeux majeurs :

L'industrie des équipements électroniques doit faire face à des défis de miniaturisation, d'intégration de multiples fonctions hétérogènes en $3 \mathrm{D}$, d'allègement et de fiabilisation des produits. Il est aussi et surtout question de réduire de façon drastique les opérations d'assemblage,

La plasturgie doit renforcer sa compétitivité en agrégeant une nouvelle forme de valeur ajoutée, en développant de nouveaux matériaux compatibles avec un processus d'intégration électronique et en repensant le flot de conception de façon à intégrer de nouvelles fonctions aux produits finis,

Le milieu de la mécanique (également vrai pour celui de la chimie, de l'automatisme,...) constitue le liant et une passerelle indéniable à la réussite des précédents acteurs. Ceux-ci doivent adapter leurs outils et méthodes de travail pour assurer la compatibilité et la viabilité de ces nouvelles méthodes de conception.

Seule une avancée conjointe permettra d'apporter et d'engranger un savoir-faire innovant pour converger vers des solutions pertinentes et optimales. 
Le cycle même de fabrication d'objets plastroniques impose, comme nous l'illustrons sur la figure 5, une formation transdisciplinaire. Cette dernière s'adressera à des problématiques multi-physiques et multi-domaine de conception et d'intégration. Elle permettra aux électroniciens comme aux plasturgistes de se comprendre mutuellement.

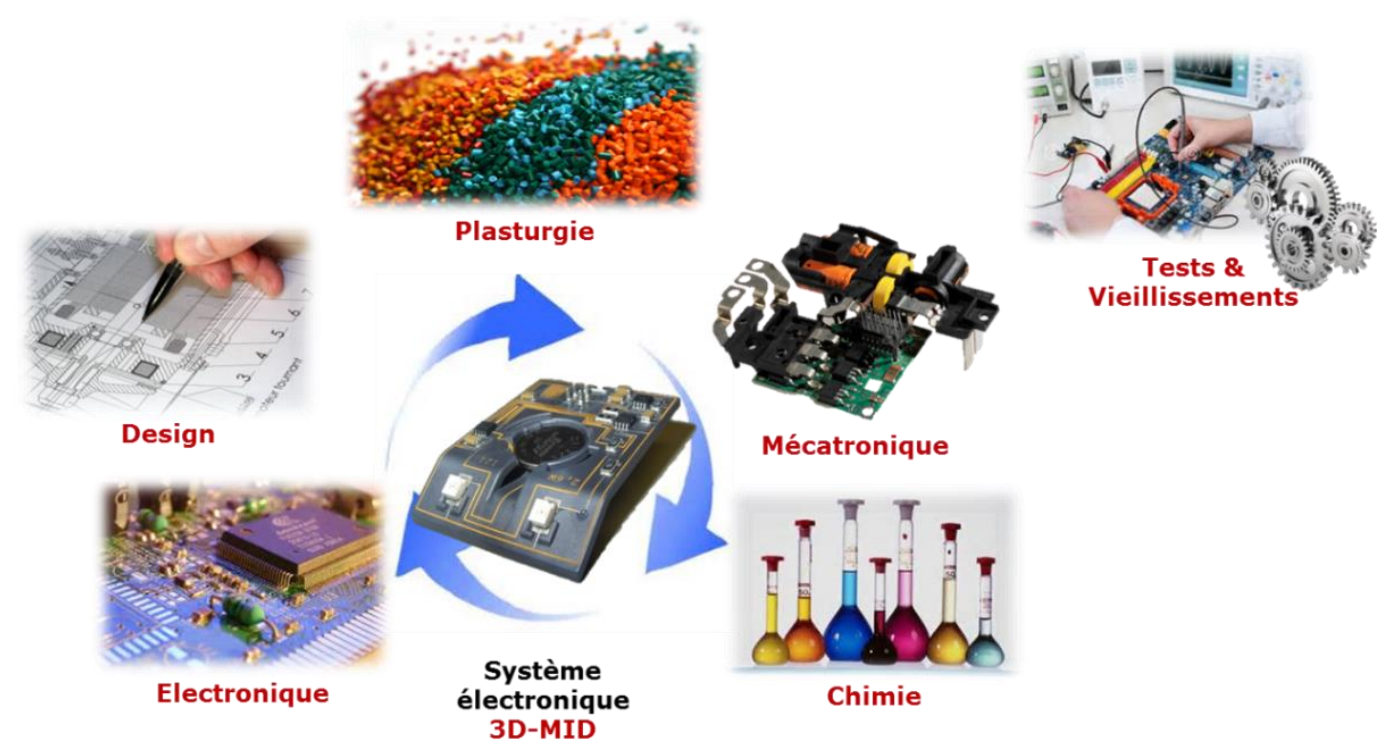

Fig. 5 : Multi-physique et multi-domaine, seul un travail conjoint et transversal permettra d'anticiper et d'optimiser au mieux les produits 3D-MID de demain (banque d'images web).

Un des buts affichés est de proposer des structures concrètes d'étude. Les formes étudiées (courbes, encombrement...) présenteront de réelles difficultés d'intégration auxquelles les industriels sont aujourd'hui confrontés. Il en sera de même dans le choix des composants et autres capteurs électroniques employés, tout comme dans les stratégies de placement.

La figure 6 montre un exemple de boucle pédagogique complète : de la conception à la réalisation, en passant par les phases de caractérisation. Cette boucle modulable pourra être étoffée en interaction avec les aspects design (au sens large) et plasturgie.

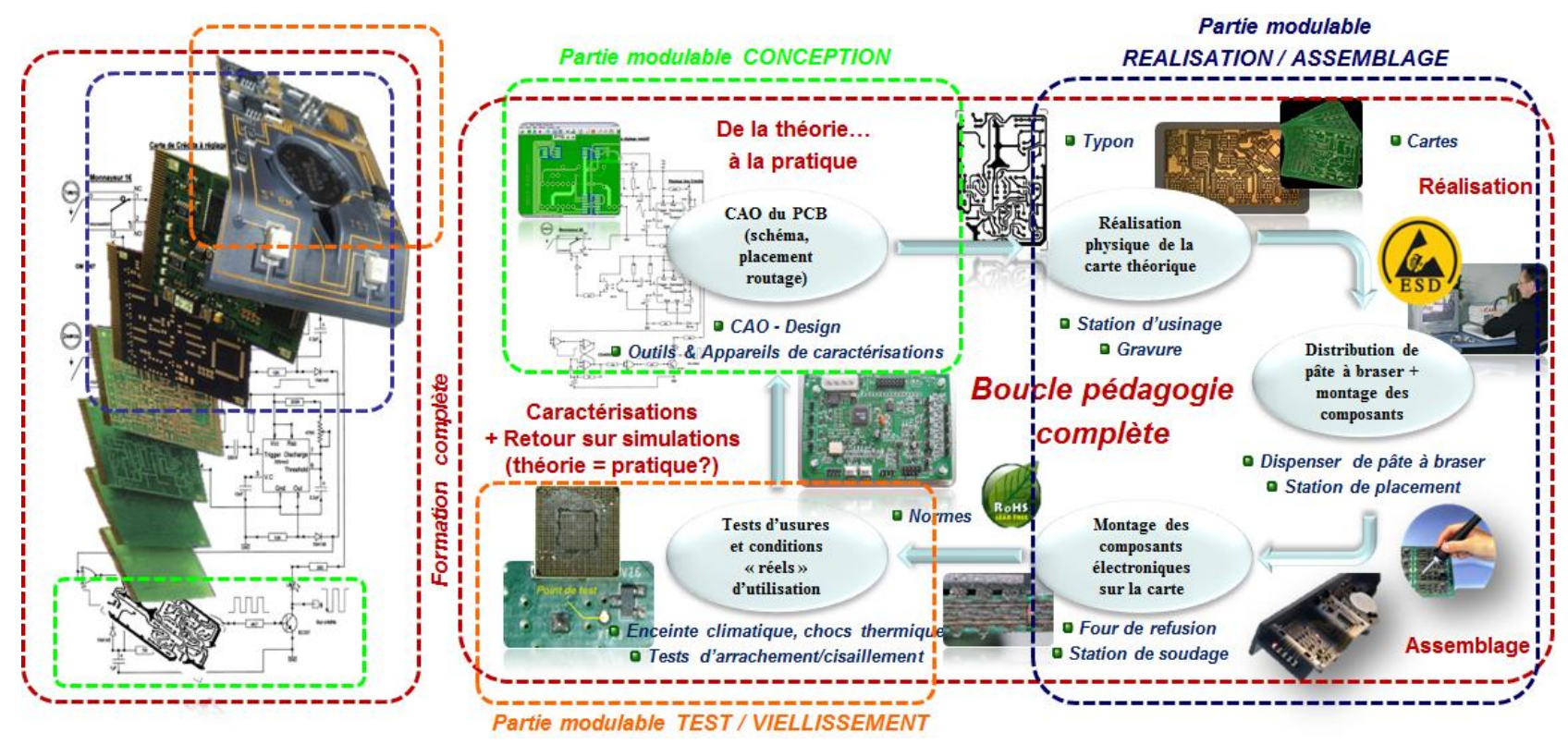

Fig. 6 : Exemple de modularité de l'enseignement proposé : Techniques de réalisation et de conception de circuits électroniques, technologies THT, CMS vers les nouvelles technologies 3D-MID (banque d'images web). 
Nous souhaiterions aborder les aspects suivants (Fig. 7) :

Conception Assistée par Ordinateur (CAO) : création d'un moule d'injection, usinage et/ou impression d'objets $3 \mathrm{D}$ en polymère,

Routage «PCB » des pistes électriques grâce à une méthode de $\mu$-tamponnage, par laser, par impression,

Métallisation des pistes électriques par l'intermédiaire de méthodes électrochimiques,

Report de composants THT et CMS sur support 2D et 3D par brasage ou collage,

Test et validation électrique à l'aide d'outils et d'instruments de caractérisation + Tests de vieillissement et contraintes mécaniques diverses.

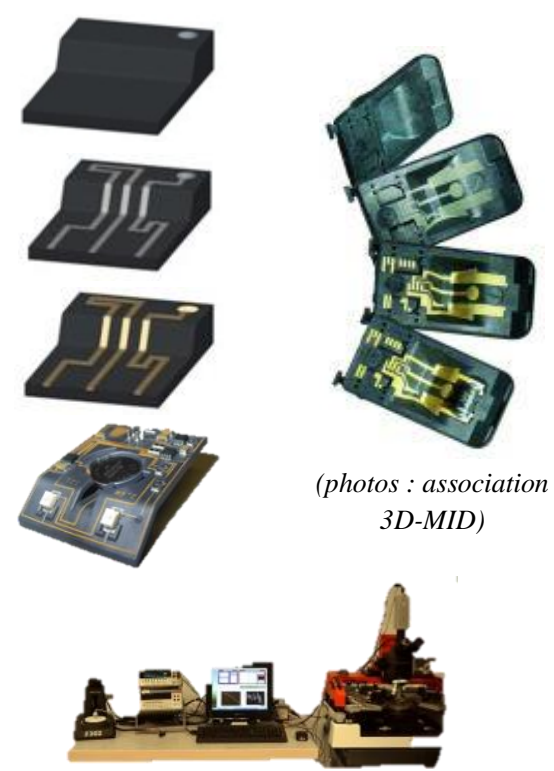

Fig. 7 : Etapes de la formation pour la réalisation d'objets $3 D$-MID

(banque d'images web).

Comme le montre la figure 8, nous proposons, par exemple, de travailler sur l'intégration de systèmes autour de la thématique du véhicule. Nous pourrions très facilement transposer ce travail autour d'autres thématiques comme par exemple l'habitat (domotique), la robotique (drone)...

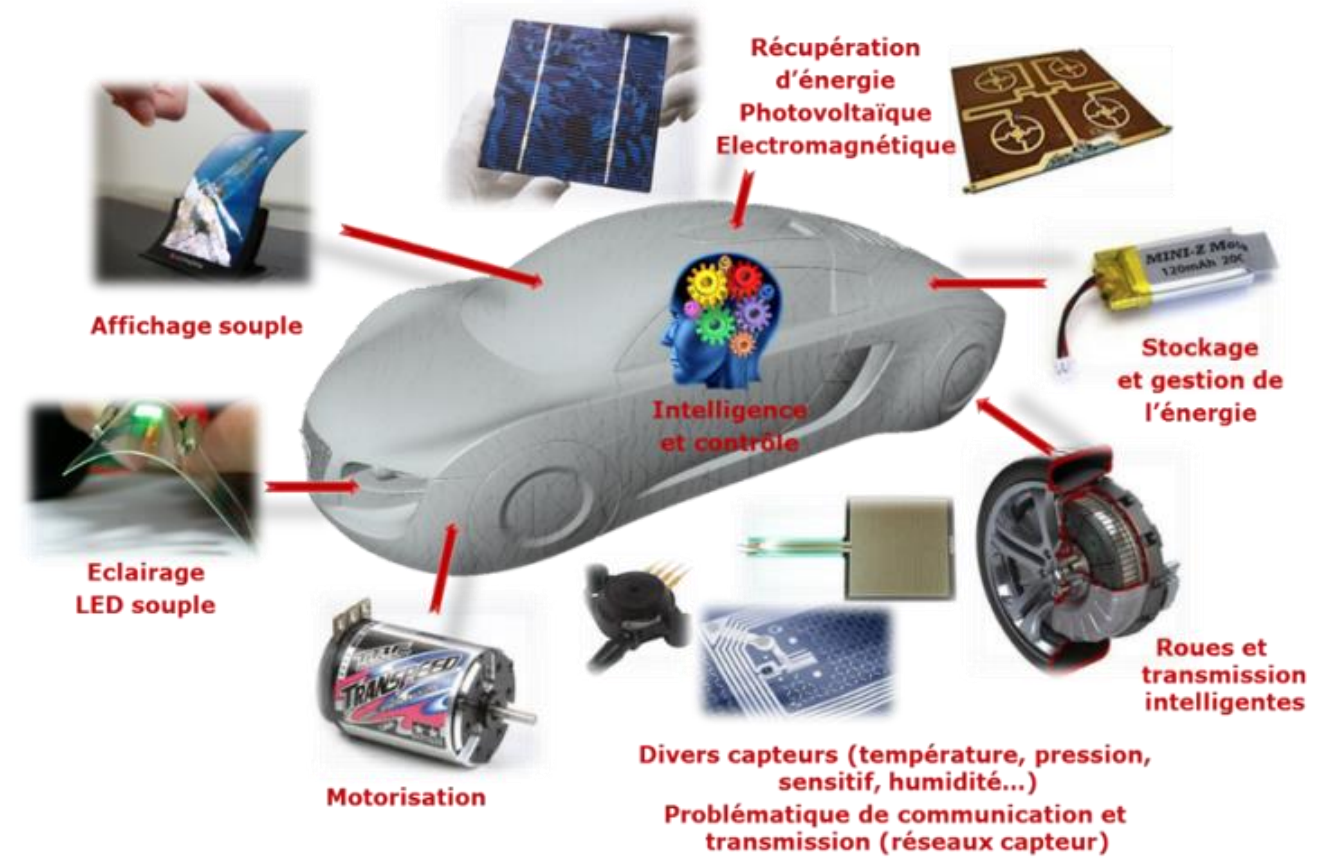

Fig. 8 : Illustration de différents capteurs, actionneurs et systèmes de commande et de gestion étudiables grâce aux technique 3D-MID autour de la thématique du véhicule (banque d'images web).

Une telle thématique permet, de surcroit, de mettre en lien l'ensemble des autres enseignements (capteurs, électronique de puissance, automatisme, programmation...) de manière à étudier et dimensionner les composants (énergie, capteurs...) à implémenter sur le système. 


\section{Public Concerne, Insertion Professionnelle}

Notre volonté est d'ouvrir le plus largement possible cette formation. Nous envisageons plusieurs cas de figure, que ce soit pour le public concerné, la durée ou le déroulement des séances. Aussi, cette formation s'adresse :
aux étudiants en formations initiale ou par alternance (Ecoles d'ingénieur, IUT, BTS, Licence et Master ; plusieurs parcours sont visés),
$\checkmark \quad$ aux professionnels en formation continue,
$\checkmark \quad$ à toute personne ayant des connaissances rudimentaires en électronique.

Ce projet vise à développer et à renforcer les compétences théoriques et pratiques des apprenants pour le développement et le prototypage de CI dans le domaine du génie électrique. Il s'inscrit dans une démarche pratique de type industrielle afin que les étudiants soient confrontés à des réalités concrètes lors d'une réalisation. L'intégration électronique sera effectuée avec des technologies de pointe aujourd'hui incontournables.

En effet, une majorité des secteurs d'activité industrielle (Biologie, Médecine, Chimie, Agroalimentaire, Design, Génie Electrique et Mécanique) implémentant des fonctions électroniques sont concernés par ces nouvelles technologies.

Cette formation vise aussi à donner des bases solides à des professionnels de corps de métiers différents (ex. électronicien et plasturgiste). Les différences d'approches, de vocabulaire, de techniques et bien d'autres compétences spécifiques, sont souvent sources à de nombreuses incompréhensions de part et d'autre. Ces mésententes impactent directement la conduite d'un projet : retard, perte de temps, surcoût pour recommencer certaines tâches, systèmes sous-optimaux... Il est donc impératif de travailler sur ces aspects et d'inculquer un « vocabulaire » compréhensible par les 2 parties.

\section{MOYENS ACTUELS}

La figure 9 illustre un certain nombre d'appareils et instruments que nous avons pu acquérir.

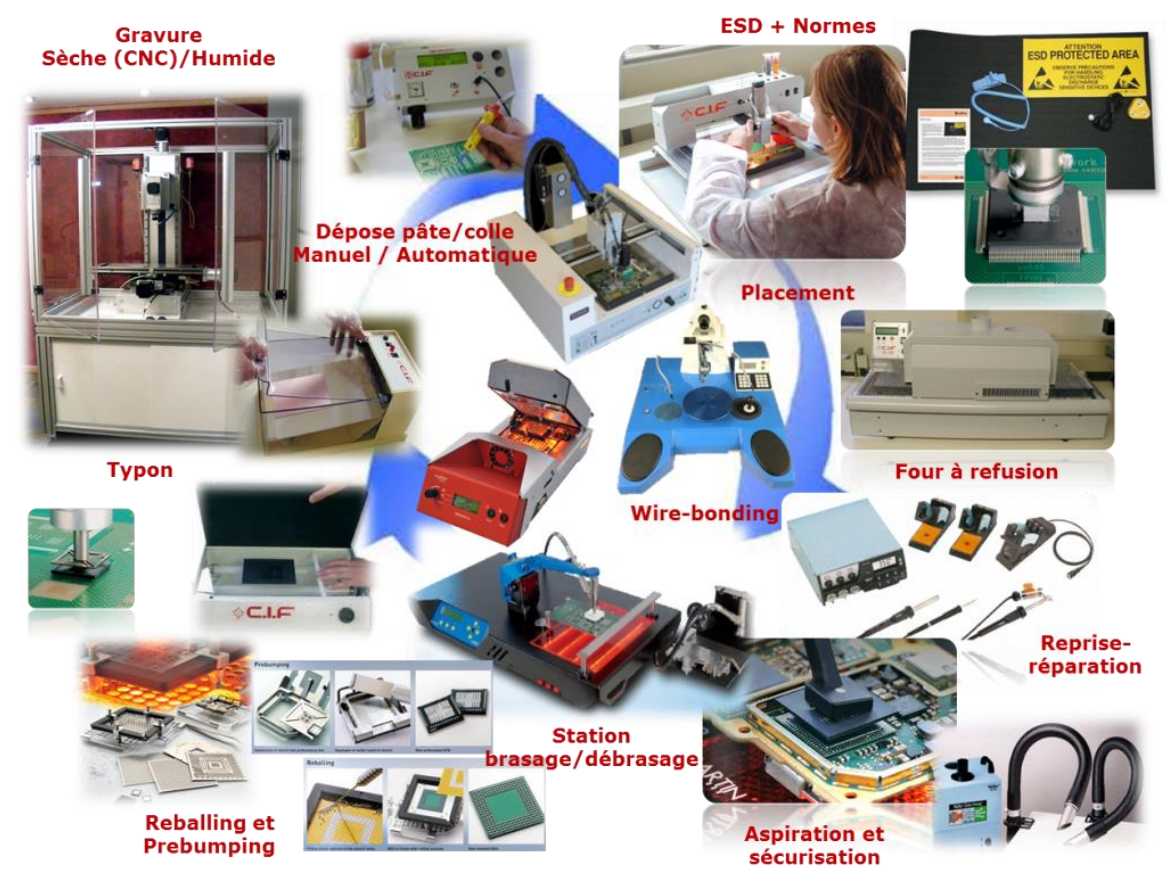

Fig. 9 : Illustration d'une partie du matériel aujourd'hui disponible pour la formation (banque d'images web).

L'ensemble de ces outils et appareils sera utilisé dans le cadre de cette formation et ouvert à la mutualisation. 
Un groupe de travail, animé par la Fondation pour l'Université de Lyon, a été constitué afin de mettre en place un pack de formation. Il est constitué d'acteurs nationaux et rhônalpins des secteurs industriels, académiques ainsi que des représentants syndicaux des branches ${ }^{2}$ issues l'électronique et de la plasturgie.

Le groupe de travail affine actuellement ses connaissances des besoins auprès d'entreprises ${ }^{3}$ qui mettent déjà en œuvre ces technologies ou qui pourraient être intéressées à le faire.

Le vivier industriel et académique Rhône-Alpin est aujourd'hui prêt à déployer ce projet en collaboration avec des partenaires nationaux. La motivation et l'implication affichées par les industriels sont un atout indéniable pour placer les apprenants en stage et faciliter leur insertion professionnelle future.

Ce projet a été labellisé par le pôle de compétitivité Plastipolis.

Nous travaillons aujourd'hui sur un calendrier nous permettant de proposer un début d'offre pour la rentrée universitaire 2016. Nous espérons une rapide déclinaison de cette offre (Licence, Master, formations continue, alternance...).

Toutes les collaborations, opportunités conjointe de travaux... sont les bienvenues.

\section{LES AUTEURS}

\section{Philippe Lombard}

Docteur en optique et radiofréquence de l'Université Joseph Fourier (UJF) de Grenoble en 2007. Il est depuis 2009 enseignant-chercheur à l'Université Claude Bernard (UCBL-Lyon1). Ses activités de recherche, à l'Institut de Nanotechnologies de Lyon (INL), portent actuellement sur les méthodes et outils de conception et d'intégration des technologies non-conventionnelles 3D-MID. En lien avec ce projet, il vient de créer une Unité d'Enseignement (UE) à destination des étudiants de Licence 3, relatif au prototypage de circuits imprimés conventionnels. Cette formation de l'UCBL débutera à la rentrée universitaire 2016.

\section{Michel Cabrera}

Ingénieur de l'Ecole Supérieur d'Electricité (1984), M. Cabrera a obtenu son doctorat en 1986 en Génie des Procédés (Institut National Polytechnique de Lorraine). Il est chercheur au CNRS depuis 1986 et a effectué une partie de sa carrière dans l'industrie de 1989 à 1996 sur les imprimantes $3 D$ (Spectra Physics / Ciba Geigy, EOS / Zeiss). Il travaille actuellement à l'Institut des Nanotechnologies de Lyon. Ses activités de recherche portent sur le développement de procédés d'élaboration de DIMs ainsi que sur d'autres procédés non conventionnels de fabrication.

\section{Vincent Semet}

Docteur en physique des matériaux (1994) de l'Université Claude Bernard Lyon 1. Maître de conférences à l'université LYON 1 depuis 1995, il a tout d'abord travaillé dans le domaine de l'émission électronique de champ ce qui l'a conduit à publier plus de 60 articles dans ce domaine. Intégrant l'institut Institut des Nanotechnologies de Lyon en 2011, son activité de recherche porte sur l'étude, le développement, et la réalisation de MID par des procédés non conventionnels.

\footnotetext{
2 Université Lyon 1, INSA, CPE-Lyon, Plastipolis, Minalogic, PEP, Alizé Plasturgie... D’autres partenaires sont susceptibles de rejoindre ce groupe (académiques, industriels, organismes de valorisation et régionaux) sans compter le soutien de plusieurs syndicats professionnels.

Ce groupe est animé par la Fondation pour l'Université de Lyon.

3 Plasturgistes fournissant l'industrie électronique, concepteurs de systèmes électroniques, sous-traitants en traitement de surface, chimistes, éditeurs de logiciels,...
} 


\section{REMERCIEMENTS}

Les auteurs souhaitent vivement remercier l'ensemble des acteurs en lien avec ce projet ${ }^{2}$, en particulier la Fondation pour l'Université de Lyon et le pôle de compétitivité Plastipolis.

Il en est de même concernant l'Université Claude Bernard Lyon 1 (UCBL) et le département Génie Electrique et des Procédés (GEP), la Faculté des Sciences et Technologies (FST) ainsi que tous les membres et acteurs du groupe de travail que nous ne pouvons citer.

\section{REFERENCES BIBLIOGRAPHIQUES}

[1] M. P.-J. Albrieux, Revue N48, «Quand l'enseignement de l'électronique rime avec Titanic», «ElectroniqueS », avril 2014.

[2] Communiqué de presse de l'APIE, «L'ENSEIGNEMENT DE L'ELECTRONIQUE EN PERDITION ou la chronique d'une catastrophe annoncée », 17 mars 2014.

[3] Rapport complet sous l'initiative de l'ASCIEL et publié sur le site du GFIE, M. Bismuth B de B consulting, «Etude sur les PCB », juillet 2014.

[4] K. Cheval, J. Coulm, S. Gout, Y. Layouni, P. Lombard, D. Léonard, F. Bessueille, V. Semet, M. Cabrera, "Progress in the manufacturing of molded interconnected devices by 3D Microcontact Printing ", Advanced Materials Research, Vol 1038 (2014) pp 57-60

[5] M. Cabrera, F. Bessueille, S. Gout, K. Cheval, J. Coulm, D. Léonard, Y. Layouni, V. Semet, « 3D Microcontact Printing for Molded Interconnect Devices », Proceedings of the $10^{\text {th }}$ International Congress Molded Interconnect Devices, Nuremberg-Fuerth, Germany, 19-20 September 2012.

[6] S. Gout, F. Bessueille, M. Moguedet, Y. Layouni, A. Errachid, D. Leonard, V. Semet, M. Cabrera, « Potential Applications of Microcontact Printing for Molded Interconnected Devices », Proceedings of the $9^{\text {th }}$ International Congress Molded Interconnect Devices, Nuremberg-Fuerth, Germany, 29-30 Sept. 2010.

[7] S. Gout, Y. Layouni, F. Bessueille, D. Leonard, A. Errachid, V. Semet, M. Cabrera, «Unconventional Selective Metallization for Polymer Microsystems », French Symposium on Emerging Technologies for Micronanofabrication, Journées Nationales sur les Technologies Emergentes en Micro-nanofabrication JNTE 10, 2426 novembre 2010, Ecole Polytechnique, Palaiseau.

\section{LISTE DE SITES INTERNET INTERESSANTS}

http://www.fondation-pour-universite-lyon.org/

http://www.plastipolis.fr/

http://inl.cnrs.fr/

http://www.univ-lyon1.fr/

http://www.poleplasturgie.net/

http://s-2p.com/

http://www.3d-mid.de/2/home/home.html

\section{TABLES ET Figures}

Fig. 1 : Evolution des technologies de câblage des cartes électroniques.

Fig 2 : (a) Tag RFID composé d'une puce silicium et d'une antenne (http://skyrfid.com/RFID_Item_Tag.php). (b) Motifs en or sur un substrat en polymère polytéréphtalate d'éthylène (PET) pour l'électronique organique (A. Benor et al. Org. Electron. 2010). (c) Transistors organiques déposés sur un substrat étirable en polydimethylsiloxane (T. Sekitani et al. Adv. Mater. 2010).

Fig. 3 : Exemples de DIMs produits en masse (article de l'Usine Nouvelle de mai 2012)

Fig. 4 : Coupe d'un Circuit Imprimé - CI multicouches + circuits empilés (BGA, flip-chip...), circuit souple (Flex) et circuit 3D-MID.

Fig. 5 : Multi-physique et multi-domaine, seul un travail conjoint et transversal permettra d'anticiper et d'optimiser au mieux les produits $3 D-M I D$ de demain.

Fig. 6 : Exemple de modularité de l'enseignement proposé : Techniques de réalisation et de conception de circuits électroniques, technologies THT, CMS vers les nouvelles technologies 3D-MID.

Fig. 8 : Illustration de différents capteurs, actionneurs et systèmes de commande et de gestion étudiables grâce aux technique 3D-MID autour de la thématique du véhicule.

Fig. 9 : Illustration d'une partie du matériel aujourd'hui disponible pour la formation. 